\title{
Environmental health equity: moving toward a solution-oriented research agenda
}

\author{
Ami R. Zota ${ }^{1}$ Bhavna Shamasunder ${ }^{2}$ \\ Received: 14 April 2021 / Revised: 15 April 2021 / Accepted: 16 April 2021 \\ (c) The Author(s), under exclusive licence to Springer Nature America, Inc. 2021
}

Health equity is the state in which everyone has the opportunity to attain full health potential and no one is disadvantaged from achieving this potential because of social position or any other socially defined circumstance [1]. Currently, the burdens of disease and poor health and the benefits of well-being and good health are inequitably distributed both in the United States and on the global landscape. This inequitable distribution is driven by social, environmental, economic, and structural factors that shape health and are themselves distributed unequally.

Increasingly, exposure scientists and epidemiologists are using multidisciplinary approaches to understand the uneven distribution of environmental exposures, links to unequal burdens of disease, and connections to socialstructural factors such as racism, racial segregation, and environmental/economic policies [2, 3]. The current global COVID-19 disease pandemic exemplifies how structural inequities can amplify disease burdens in vulnerable groups. Collectively sustained effort in this arena, and deepening this body of knowledge can move us toward health equity and help secure environmental and health justice.

Environmental health equity is a growing and critical area of the environmental health sciences. The exposure science and environmental epidemiology communities have long focused on characterizing the disproportionate burden of environmental harm among marginalized communities, and consequent health impacts using a range of methods from large-scale epidemiologic studies to citizen science approaches.

$\triangle$ Ami R. Zota

azota@email.gwu.edu

1 Department of Environmental and Occupational Health, George Washington University Milken Institute School of Public Health, Washington, DC, USA

2 Department of Urban and Environmental Policy and Public Health, Occidental College, Los Angeles, CA, USA
In contrast, there has been less sustained attention to conceptual and empirical work to understand how structural factors-such as how structural racism and interlocking systems of oppression shape environmental and occupational landscapes, and relatedly health and wellness [4]. We have too often focused on proximate risk factors such as individual behaviors in population health studies. This focus can limit the scope of questions, and related solutions that we can leverage to make widespread gains to improve health and quality of life. New frameworks are required to move us toward environmental health equity, including improved interdisciplinary partnerships, continued focus on community-based research, and integrating root cause analyses into ongoing research efforts.

This special issue incorporates pressing issues in environmental health that span global to local, with research that spans diverse methodological approaches and frameworks. Studies such as those led by Drs. Van Horne and Williams, focus on vulnerable populations and are working to integrate structural processes (e.g., land use changes). Complex mixtures have posed an environmental justice challenge given cumulative burdens and vulnerability; Van Horne et al.'s work helps to unravel some of these ongoing challenges through an analysis of metal mixtures nearby an active industrial facility. Low-cost sensors are increasingly debated in environmental justice as one way to democratize air pollution information. deSouza et al. considers the growing field of low-cost sensor use for environmental justice, and distributional inequity in sensor network deployment that limits widespread access to these technologies.

Pregnancy outcomes and fetal development are sensitive markers of environmental perturbation and can have implications across the life course. Three papers focus on the interplay between chemical and non-chemical stressors on pregnancy outcomes, which help to advance knowledge of cumulative impacts in environmental justice communities. Mekonnen et al. observed that within urban California counties, the highest risk of preterm birth associated 
with air pollution was among mothers living in lower socioeconomic areas. Milando et al. used simulated methods to estimate birthweight changes following hypothetical environmental interventions in environmental justice communities. Huang et al. examined the role of multiple metals exposures in preterm birth among mother-offspring pairs in Bangladesh and further examined upstream socioeconomic risk factors specific to the Bangladeshi context.

Papers in this collection advance the environmental injustice of the beauty framework [5] in three important ways. These studies provide empirical data on patterns of product use to better understand and unravel disparate exposures to synthetic environmental chemicals linked to consumer products. Dodson et al. examine racial and ethnic differences in product use among diverse women, including multiracial women, in California. James Todd et al. evaluates hormonal activity data in commonly used Black hair care products in order to better understand plausible contributions to health disparities, and Wesselink et al. examine associations of personal care product use and phthalates exposures among reproductive age Black women in Detroit, Michigan.

Occupational exposures provide sentinel data for chemical vulnerability among those exposed through their workplaces. Researchers are grappling with issues related to sexism, gender justice, and occupational vulnerability for multiply marginalized populations. These papers integrate attention to intersectional identities that can impact access to information, health outcomes, and policy gaps. Curl et al. examines urinary pesticides biomarkers among Latina women farmworkers in Idaho. Their preliminary findings indicate potential for increased pesticide exposure among this group of workers, and the need for additional studies. In India, while women make up half of the total workforce in the tea plantation sector, there has been little data on their exposures and workplace protections. Venugopal et al. characterize pesticide exposures among female laborers on a tea plantation to better support preventative measures for these workers. Ingram et al. consider 50 years of OSHA and promote a socio-ecological framework for better protecting small business workers given limited attention to these workplaces in low income, communities of color.

Green development has been one pathway to reduce environmental inequalities. Werthmann et al. and Williams et al. examine the built environment as a contributor to environmental health disparities, and consider interventions at the housing and community level. Werthmann et al. compare pesticide biomonitoring data in children living in green housing to children living in non-green housing and show no significant difference among their modest sample. Williams et al.'s novel study suggest a nuanced relationship between social environmental stressors, pressure of displacement related to greenspace redevelopment, and sleep quality among Black adults.

Disasters, including natural disasters and the ongoing global pandemic, can reveal entrenched inequality and deepen existing vulnerabilities. Hurricane Harvey worsened access to immediate resources in poor communities of color but less considered is how it opened vulnerability to greater pollution during the disaster through increased toxic releases in socioeconomically vulnerable areas. The pandemic has exacerbated and deepened many inequalities and Zhang et al contribute to this through an examination of neighborhood characteristics associated with COVID-19 burden in Illinois and how these factors vary by age group. The commentary by Sasser et al calls for analyses of the intersecting patterns of inequality and systemic logics, through the case of inland California to highlight the need for an intersectional approach that addresses the compounding effects of overlapping and interdependent systems of oppression.

While these research projects and questions begin to move us in the right direction, the next generation of environmental health equity scholarship should incorporate collaborations across disciplines, and consider how research knowledge can support systemic changes, including in the arenas of policy and practice.

\section{Compliance with ethical standards}

Conflict of interest The authors declare no competing interests.

Publisher's note Springer Nature remains neutral with regard to jurisdictional claims in published maps and institutional affiliations.

\section{References}

1. National Academies of Sciences, Engineering, and Medicine'. Communities in action: pathways to health equity. Washington, DC: The National Academies Press; 2017.

2. Johnston J, Cushing L. Chemical exposures, health, and environmental justice in communities living on the fenceline of industry. Curr Environ Health Rep. 2020;7:48-57.

3. Nardone A, Casey JA, Morello-Frosch R, Mujahid M, Balmes JR, Thakur N. Associations between historical residential redlining and current age-adjusted rates of emergency department visits due to asthma across eight cities in California: an ecological study. Lancet Planet Health. 2020;4:e24-31.

4. Zota AR, VanNoy BN. Integrating intersectionality Into the exposome paradigm: a novel approach to racial inequities in uterine fibroids. Am J Public Health. 2021;111:104-9.

5. Zota AR, Shamasunder B. The environmental injustice of beauty: framing chemical exposures from beauty products as a health disparities concern. Am J Obstet Gynecol. 2017;217:418. e1-418.e6. 